\title{
A GENERALIZED GAUSS MARKOV MODEL FOR SPACE OBJECTS IN BLIND RESTORATION OF ADAPTIVE OPTICS TELESCOPE IMAGES *
}

\author{
Brian D. Jeffs $\dagger$, Sheila Hong $\dagger$ and Julian Christout \\ $\dagger$ Department of Electrical and Computer Engineering, 459 CB, Brigham Young University, Provo, UT 84602, \\ phone: (801)378-3062, FAX: (801) 378-6586, email: bjeffs@ee.byu.edu \\ $\ddagger$ Starfire Optical Range, Air Force Research Laboratory AFRI,/DES
}

\begin{abstract}
This paper introduces a blind method based on Bayesian maximum a posteriori estimation theory for restoring images corrupted by noise and blurred by one or more unknown point spread functions. Image and blur prior information is expressed in the form of parametric generalized Gauss Markov random field models. A method for estimating the GGMRF neighborhood influence parameters is presented, along with examples of blind restoration to reduce residual blur in adaptive optics telescope images of space objects.
\end{abstract}

\section{INTRODUCTION}

In this paper we consider the blind multiframe restoration problem of recovering images corrupted by noise and blurred by one or more unknown point spread functions (psf). The Bayesian maximum a posteriori (MAP) estimation approach is well suited to blind restoration because it provides a framework for expressing prior information about both the image and unknown blur. Using appropriate probability density function (pdf) models as image and blur priors enables us to overcome the inherent ambiguity of the blind inverse problem, and serves to regularize the solution. We will show that with correct parameter selection, the generalized Gauss Markov random field (GGMRF) model [1] is effective for both prior pdf's. The GGMRF model is able to represent a wide range of practical image types, including both structured, hard edged fields typical of the true image, and smooth, "low pass" or "band limited" images typical of blurring psf's.

Though the algorithm presented here may be used in a variety of blind deconvolution applications, we are particularly interested in high resolution restoration of multiframe adaptive optics (AO) telescopic images. AO systems can remove much of the atmospheric-turbulence-induced blur in astronomical and orbiting space objects imagery, but there is typically some adaptation error [2]. Though the general structure of this residual blur is known [3], the detail is not, and varies significantly over a period of milliseconds. A sequence of $\mathrm{AO}$ images of a single object provides multiple observations with different unknown psf's, and constitutes a multiframe, blind image restoration problem.

*SPONSORED BY THE AIR FORCE OFFICE OF SCIENTIFIC RESEARCH, BOLLING AIR FORCE BASE, DC.
We adopt the following image observation model for both single and multiple frame data representation

$$
\begin{aligned}
\overline{\mathbf{g}} & =\mathcal{H} \mathbf{f}+\bar{\eta}, \text { where } \\
\overline{\mathbf{g}} & =\left[\mathbf{g}_{1}, \mathbf{g}_{2}, \cdots, \mathbf{g}_{M}\right]^{T}, \quad \bar{\eta}=\left[\eta_{1}, \eta_{2}, \cdots, \eta_{M}\right]^{T} \\
\mathcal{H} & =\left[\mathbf{H}_{1}^{T}, \cdots \mathbf{H}_{M}^{T}\right]^{T}
\end{aligned}
$$

where $M$ is the number of frames, $\mathbf{g}_{i}, \mathbf{f}$, and $\eta_{i}$ are vectors formed by column scanning the 2-D images of the $i^{\text {th }}$ observation frame, the true image, and the $i^{t h}$ noise frame respectively. $\mathbf{H}_{i}$ is the doubly block Toeplitz convolution matrix formed from the $i^{i, h}$ frame $\mathrm{psf}, \mathbf{h}_{i}$.

\section{PROBLEM FORMULATION}

Assuming $\mathbf{f}$ and $\mathcal{H}$ are statistically independent (a reasonable assumption in practice), the blind MAP restoration problem may be stated as

$$
\hat{\mathbf{f}}, \hat{\mathcal{H}}=\arg \max _{\mathbf{f}, \mathcal{H}} p_{g \mid f, h}(\overline{\mathbf{g}} \mid \mathbf{f}, \mathcal{H}) p_{f}(\mathbf{f}) p_{h}(\mathcal{H})
$$

In order to solve equation (2), we adopt the following probability density function models. $p_{\eta}(\bar{\eta})$ is assumed to be zero mean, i.i.d. Gaussian. This implies that $p_{g \mid f, h}(\overline{\mathbf{g}} \mid \mathbf{f}, \mathcal{H})$ is i.i.d. Gaussian with a mean of $\mathcal{H}$ f. The image is modeled as a GGMRF, with pdf given by the Gibbs distribution [1]

$$
p_{f}(\mathbf{f})=\frac{1}{Z_{f}} \exp \left\{-\beta \sum_{<s, t>\in \mathcal{C}_{f}} b_{s, t}\left|f_{s}-f_{t}\right|^{p}\right\}
$$

where $\mathcal{C}_{f}$ is the set of pixel cliques invoked by the specified neighborhood system, $p$ is the GGMRF shape parameter, $S_{f}$ is the set of all pixels in the image lattice, $f_{s}$ is the $s^{t h}$ pixel in $\mathrm{f}$, and $b_{s, t}$ are neighborhood influence weights.

The blur pdf is also modeled as a GGMRF

$$
\begin{aligned}
p_{h}(\mathcal{H}) & =\frac{1}{Z_{h}} \exp \left\{-\alpha \sum_{s \in S_{h}} d_{s}\left|h_{s}-\mu_{h, s}\right|^{q}-\right. \\
\alpha & \left.\sum_{<s, t>\in \mathcal{C}_{h}} c_{s, t}\left|\left(h_{s}-\mu_{h, s}\right)-\left(h_{t}-\mu_{h, t}\right)\right|^{q}\right\}
\end{aligned}
$$

where $\mathcal{C}_{h}$ is the set of all cliques for the blur neighborhood system, $q$ is the blur GGMRF shape parameter, $S_{h}$ is the 


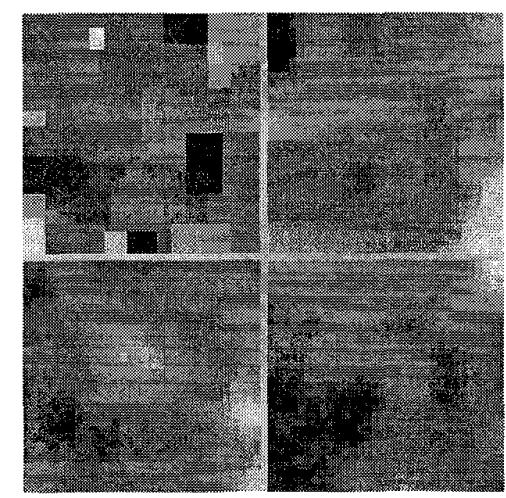

Figure 1. Synthetic GGMRF images. a) [top left] $p=0.5$, b) [top right] $p=1.0, \mathrm{c})$ [bot. left] $p=2.0, \mathrm{~d}$ ) [bot. right] $p=3.0$.

set of all points in the blur lattice over all frames, and $c_{s, t}$ and $d_{s}$ are neighborhood influence weights.

The GGMRF model is particularly flexible and useful for image restoration problems because it is capable of representing a wide variety of statistical image classes using just a few parameters. Equations (3) and (4) can be viewed as definitions for random texture fields. Model parameters control the structure of this texture, and can be chosen to represent both typical images and blurs. $p$ and $q$ are known as shape parameters, and control the "edginess," or transition structure in the image. $b_{s, t} c_{s, t}$ and $d_{s}$ jointly control the correlation structure between neighboring pixels. With appropriate value selections, the model can be parameterized to generate fields that look like the detail in a wide range of visual textures.

Figure 1 shows an example of how shape parameter $p$ (or $q$ ) affects the GGMRF texture field. The images shown were generated as synthetic Markov random fields using a first order uniform neighborhood while only $p$ was varied. We propose that Figure 1a, with $p=0.5$, is a better statistical match for the blocky geometric structures of man-made objects, like satellites, than are the other three fields. We also assert that Figure 1d, with $p=3.0$, is an good model for the deviation from the mean, $\mu_{h, s}$, for residual $\mathrm{AO}$ blur. In practice, we will set $0.5<p<1.5$ and $2.0<q<4.0$.

Including a mean, $\mu_{h, s}$, in the $p_{h}(\mathcal{H})$ model (which was not needed in $p_{f}(\mathbf{f})$ ) allows us to incorporate prior psf information from previous experiments. For example, in astronomical imaging isolated stars in a nearby field, or frames acquired on different experiments, can be averaged to serve as the reference mean. An analytic model can also be used as a mean. The Lorentzian function has been proposed as a model for mean residual AO blur [3] [4].

Substituting equations (4),(3) and our $p_{g \mid g, h}(\overline{\mathbf{g}} \mid b f f, \mathcal{H})$ model into (2), and performing a little algebra yields

$$
\begin{aligned}
\hat{\mathbf{f}}, \hat{\mathcal{H}} & =\arg \underset{\mathbf{f}, \mathcal{H}}{\min } \sum_{i=1}^{M}\left\|\mathbf{g}_{i}-\mathbf{H}_{i} \mathbf{f}\right\|^{2}+ \\
\gamma & \sum_{<s, t>\in \mathcal{C}_{f}} b_{s, t}\left|f_{s}-f_{t}\right|^{p}+\lambda \sum_{s \in S_{h}} d_{s}\left|h_{s}-\mu_{h, s}\right|^{q}+
\end{aligned}
$$

$$
\lambda \sum_{<s, t>\in \mathcal{C}_{h}} c_{s, t}\left|\left(h_{s}-\mu_{h, s}\right)-\left(h_{t}-\mu_{h, t}\right)\right|^{q}
$$

where we have taken the logarithm of the right hand side and have dropped additive constants. $\gamma$ and $\lambda$ control the relative influence as regularizing terms that the image and blur pdf's have on the solution respectively.

Equation (5) can be solved using stochastic relaxation, such as the Metropolis algorithm, when either $p$ or $q$ is less than one. An efficient steepest descent algorithm may be used when $p, q \geq 1[5]$.

\section{GGMRF PARAMETER ESTIMATION}

Shape parameter estimation for the GGMRF model is crucial for higher quality image restoration. Without appropriate parameter settings, the image and blur priors will bias the restoration solution to some nonsense result. The key model values of equation (3) which control image structure are the shape parameter, $p$, and neighborhood influence parameters, $b_{s, t}$ (corresponding parameters in equation (4) are $q$ and $\left.c_{s, t}\right)$. The value of $p$ controls image edge activity, while $b_{s, t}$ values determine image correlation structure. Though it is difficult to obtain good parameter estimates from a degraded image, it is often possible to find an ensemble of images similar to the desired true image. Estimates from this ensemble can then be used in restoring the degraded image. This ensemble approach has been used with a sample kurtosis based $p$ estimator, and has been shown to produce better restoration results than when an arbitrary $p$ value is used [6] [7].

In this section we demonstrate the effectiveness of the coding method for GGMRF neighborhood influence parameter estimation. We will show that the maximum likelihood (ML) coding estimator for Gauss Markov random fields (GMRF) works surprising well when applied to GGMRF images. This is true even though the method is not a true ML estimator in the GGMRF case.

The conditional density function for a Gauss Markov random field can be expressed in a form similar to the GGMRF model of equation (3),

$$
p\left(f_{s} \mid f_{t}, \forall t \in \delta_{s}\right)=\frac{1}{Z_{f}} \exp \left\{\sum_{t \in \delta_{s}} b_{s, t}\left|f_{s}-f_{t}\right|^{2}\right\},
$$

where $\delta_{s}$ is the neighborhood of $s$. It can be shown [8] that this representation is equivalent to a form used by Kayshap, Chellappa and others [9]

$$
p\left(f_{s} \mid f_{t}, \forall t \in \delta_{s}\right)=\frac{1}{\sqrt{2 \pi \sigma^{2}}} \exp \left\{-\frac{1}{2 \sigma^{2}}\left[f_{s}-\sum_{t \in \delta_{s}} \theta_{t} f_{t}\right]^{2}\right\} \text {. }
$$

The advantage of equation (7) is that well known techniques are available for estimating $\theta_{t}$. The relationship between $\theta_{t}$ and our neighborhood influence parameters, $b_{s, t}$, is $\theta_{t}=\frac{b_{s, t}}{\sum_{t \in \delta_{s}^{\prime}}^{b_{s, t}}}$. Thus, given a method to estimate $\theta_{t}$, we can use it to estimate $b_{s, t}$ in a GMRF.

The coding method was introduced by Besag [10], and later updated by Kashyap and Chellappa [9]. We adopt the 
Chellappa notation in this discussion. The main idea of the coding method is to separate the lattice set $S$ into several disjoint sets, $S^{(k)}$, called codings, such that no two sites in one $S^{(k)}$ are neighbors, and where $S=S^{(1)} \cup S^{(2)} \cup \cdots S^{(k)}$. Figure 2 shows the codings for a first-order system.

\begin{tabular}{|l|l|l|l|l|}
\hline 1 & 2 & 1 & 2 & 1 \\
\hline 2 & 1 & 2 & 1 & 2 \\
\hline 1 & 2 & 1 & 2 & 1 \\
\hline 2 & 1 & 2 & 1 & 2 \\
\hline 1 & 2 & 1 & 2 & 1 \\
\hline
\end{tabular}

Figure 2. Coding for the Four-neighborhood (First-order) System. All pixels designated with the same number belong to a single coding.

Since within a single coding no pixels are neighbors, and thus their conditional distributions are independent, we may write

$$
p\left(f^{(k)} \mid \theta\right)=\prod_{s \in S^{k}} p\left(f_{s}^{(k)} \mid f_{t}, \forall t \in \delta_{s}, \theta\right)
$$

where all $f_{s}^{(k)}$ on the neighborhood are mutually independent, and $\theta=\left[\theta_{1}, \theta_{2}, \cdots, \theta_{|S|}\right]^{T}$. Maximizing equation (8) with respect to $\theta$ gives the ML coding estimate, $\widehat{\theta^{k}}$. The arithmetic average of the estimate over all codings yields the final value of $\widehat{\theta}$.

For a first order zero mean GMRF, the coding estimate for $\widehat{\theta}^{k}$ is the solution, with respect to $\theta$, of the linear system

$$
\begin{aligned}
& \Lambda \theta=\chi \text {, where } \\
& \Lambda=\left[\begin{array}{ll}
\sum_{s} f_{s+r_{1}}^{2} & \sum_{s} f_{s+r_{1}} f_{s+r_{2}} \\
\sum_{s}^{s} f_{s+r_{2}} f_{s+r_{1}} & \sum_{s} f_{s+r_{2}}^{2} \\
\sum_{s}^{s} f_{s+r_{3}} f_{s+r_{1}} & \sum_{s} f_{s+r_{3}} f_{s+r_{2}} \\
\sum_{s} f_{s+r_{4}} f_{s+r_{1}} & \sum_{s} f_{s+r_{4}} f_{s+r_{2}}
\end{array}\right. \\
& \left.\begin{array}{ll}
\sum_{s} f_{s+r_{1}} f_{s+r_{3}} & \sum_{s} f_{s+r_{1}} f_{s+r_{4}} \\
\sum_{s} f_{s+r_{2}} f_{s+r_{3}} & \sum_{s} f_{s+r_{2}} f_{s+r_{4}} \\
\sum_{s} f_{s+r_{3}}^{2} & \sum_{s} f_{s+r_{3}} f_{s+r_{4}} \\
\sum_{s} f_{s+r_{4}} f_{s+r_{3}} & \sum_{s}^{s} f_{s+r_{4}}^{2}
\end{array}\right] \text {, } \\
& \theta=\left[\theta_{1}, \theta_{2}, \theta_{3}, \theta_{4}\right]^{T} \text {, } \\
& \chi=\left[\begin{array}{l}
\sum_{s} f_{\left(s+r_{1}\right)} f_{(s)} \\
\sum_{s} f_{\left(s+r_{2}\right)} f_{(s)} \\
\sum_{s}^{s} f_{\left(s+r_{3}\right)} f_{(s)} \\
\sum_{\left(s+r_{4}\right)} f_{(s)}
\end{array}\right],
\end{aligned}
$$

and where $f \in S^{(k)}$. In this equation we have used a relative position index, $r_{n}$, to neighbor pixel $t=s+r_{n}$. For a firstorder neighborhood, $r_{n}=\left\{r_{0}=(0,0), r_{1}=(0,1), r_{2}=\right.$ $\left.(0,-1), r_{3}=(-1,0), r_{4}=(1,0)\right\}$. This is illustrated in Figure 3 .

ML estimation of $b_{s, t}$ for a GGMRF with $p \neq 2$ is an unsolved problem because the partition function, $Z_{f}$, depends on these parameters in an unknown way that cannot easily be evaluated. Lacking a direct solution, we hypothesize

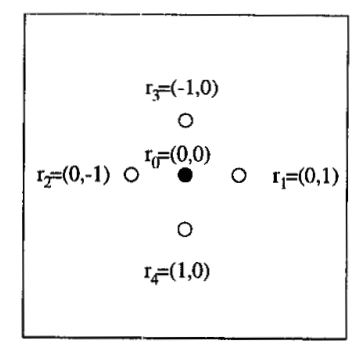

Figure 3. First-Order Neighborhood System For the Coding Method.

that the ML coding estimator for neighborhood influence parameters in a GMRF is an acceptable approximation for the corresponding parameters in a GGMRF for $p \approx 2$. The remainder of this section presents an experimental evaluation of this hypothesis. It is shown that for $0.8 \leq p \leq 2.6$, the first-order GMRF coding estimate of equation (9) provides surprisingly accurate estimates of $b_{s, t}$ for a GGMRF.

The experimental procedure involved generating a variety of synthetic GGMRF random fields with known shape and neighborhood influence parameters. Estimator results were then compared with the known values. Seven different shape parameter values, $p=0.8,1.1,1.4,1.8,2.0,2.2$, and 2.6 , (covering the range of values that we have commonly used in restoration applications), and four sets of neighborhood influence parameters were used in all possible combinations to generate the test image set. Chen's algorithm was used for image synthesis [11]. For notational convenience we define an augmented influence parameter matrix

$$
\mathcal{B}=\left[\begin{array}{l}
\theta_{1}, r_{1} \\
\theta_{2}, r_{2} \\
\theta_{3}, r_{3} \\
\theta_{4}, r_{4}
\end{array}\right] .
$$

Figure 4 shows an example of the four different images generated using different influence parameters $\left(\mathcal{B}_{1} \cdots \mathcal{B}_{4}\right)$ as defined in Table ?? while $p$ is fixed at 1.1.

Table 1 presents the estimation results for the $p=1.1$ case. Note that even though $p$ is much different than the GMRF value of $p=2.0$, each estimated influence parameter (weight) was correct to within 0.02 . This was the maximum estimation error over all trials, for all combinations of $p$ and $\mathcal{B}$. These results demonstrate that the proposed coding method, though designed for GMRF images, is an excellent neighborhood influence parameter estimator for GGMRF images.

An example of blind restoration using estimated neighborhood influence parameters is presented in Section 4 ..

\section{SPACE OBJECT RESTORATION EXAMPLES}

This section presents exanaples of GGMRF blind restoration of a satellite and an asteroid image degraded by $\mathrm{AO}$ residual blur. These results demonstrate the potential of the new method for high resolution recovery of space objects in partially corrected $\mathrm{AO}$ multiframe sequences. 

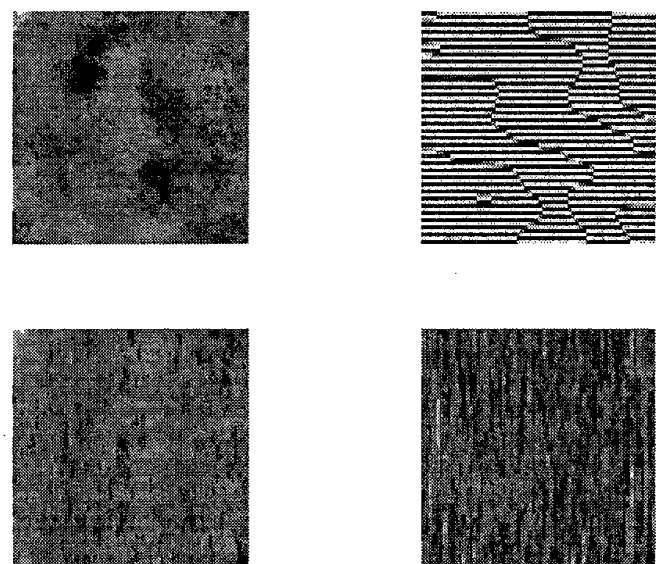

Figure 4. Synthetic GGMRF Images for Shape Parameter $p=1.1$. a) [Top Left] true neighborhood influence matrix, $\mathcal{B}_{1}$, b) [Top Right] $\mathcal{B}_{2}$, c) [Bottom Left] $\mathcal{B}_{3}$, d) [bottom Right] $\mathcal{B}_{4}$.

For the first example, a series of five partially corrected AO star images were used as the blurring psf's, and noise was added at a level of $20 \mathrm{~dB}$ peak SNR. Each observed frame is 64 by 64 pixels. Figure 5 shows the true image at high resolution (128 by 128 pixels), one of the five observed frames for reference, and the steepest descent algorithm result. GGMRF models were used for both the object and the psf's, with $p=1.1$ and $q=2.5$. The object model was first order, while the blur psf model was second order to impose more smoothness in blur estimates. Note that though the solution is noisy due to low observation SNR, much of the satellite structure is recovered. Figure 6 shows the blur estimates corresponding to Figure 5 . It is noteworthy that the central peak structure of the psf's are correctly estimated, though there is error in the random halo structure due to the low SNR. The low level structure has been emphasized in this figure by using a logarithmic grayscale.

The second example demonstrates use of estimated neighborhood influence parameters in restoring an image of asteroid Ida obtained by a spacecraft close fly-by. The original image is shown in Figure 7a. Three distinct AO residual blurs were synthesized using randomized realizations of Drummond's Lorentzian model [3], and then used to blur Figure 7a. Figure $7 \mathrm{~b}$ shows one of the three observed frames, generated by convolving the original image with one frame of the blur model. Figure $7 \mathrm{c}$ is the GGMRF restoration result obtained with a first-order uniform neighborhood model for $\mathbf{f}, p=1.1$, a second order model for $\mathbf{H}$, and $q=3.0$. Note the significant recovery of detail in Figure $7 \mathrm{c}$ as compared to $7 \mathrm{~b}$.

The original asteroid image was processed using the coding method to estimate neighborhood influence parameters. The computed values were $\theta_{1}=\theta_{2}=0.339$, and $\theta_{3}=\theta_{4}=0.164$. The restoration using these parameter values, shown in Figure $7 \mathrm{~d}$, recovers slightly more detail than the restoration using a uniform neighborhood influence parameter.

\begin{tabular}{|c|c|}
\hline $\begin{array}{c}\text { True Weight Matrix } \\
\mathcal{B}\end{array}$ & $\begin{array}{c}\text { Estimated Weight Matrix } \\
\hat{\mathcal{B}}\end{array}$ \\
\hline $\mathcal{B}_{1}=\left[\begin{array}{rr}0.250, & (1,0) \\
0.250, & (-1,0) \\
0.250, & (0,1) \\
0.250, & (0,-1)\end{array}\right]$ & {$\left[\begin{array}{rr}0.270, & (1,0) \\
0.251, & (-1,0) \\
0.247, & (0,1) \\
0.258, & (0,-1)\end{array}\right]$} \\
\hline $\mathcal{B}_{2}=\left[\begin{array}{rr}0.250, & (1,0) \\
0.250, & (-1,0) \\
-.250, & (0,1) \\
-.250, & (0,-1)\end{array}\right]$ & {$\left[\begin{array}{rr}0.258, & (1,0) \\
0.258, & (-1,0) \\
-.264, & (0,1) \\
-.263, & (0,-1)\end{array}\right]$} \\
$\mathcal{B}_{3}=\left[\begin{array}{rr}0.083 & (1,0) \\
0.083, & (-1,0) \\
0.417, & (0,1) \\
0.417, & (0,-1)\end{array}\right]$ & {$\left[\begin{array}{rr}0.080, & (1,0) \\
0.075, & (-1,0) \\
0.425, & (0,1) \\
0.432, & (0,-1)\end{array}\right]$} \\
$\mathcal{B}_{4}=\left[\begin{array}{rr}0.000, & (1,0) \\
0.000, & (-1,0) \\
0.500, & (0,1) \\
0.500, & (0,-1)\end{array}\right]$ & {$\left[\begin{array}{rr}-.006, & (1,0) \\
-.005, & (-1,0) \\
0.486, & (0,1) \\
0.500, & (0,-1)\end{array}\right]$} \\
\hline
\end{tabular}

Table 1. GGMRF Neighborhood Influence Parameter Estimation, $p=1.1$.

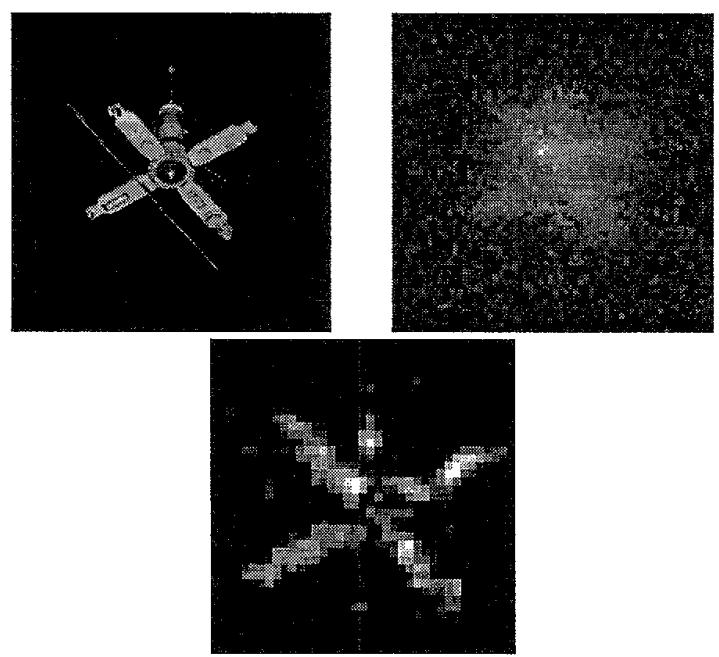

Figure 5. Steepest descent GGMRF restoration of synthetic Satellite data with $20 \mathrm{~dB}$ peak SNR. a) [left] High resolution $(128 \times 128)$ true object. b) [middle] An example observation frame $(64 \times 64)$. c) [right] Restored image, $40 \times 40$ pixel window. Parameter settings were $p=1.1, q=2.5, \gamma=0.2$, $\lambda=200, \alpha=0.001$, and 1,000 iterations. 

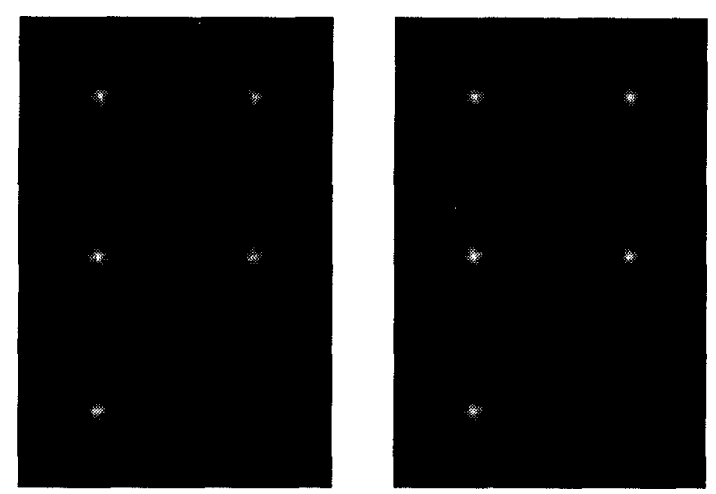

Figure 6. Blur estimates for synthetic Satellite data. a) [left] True blur psf's. b) [right] Blur estimates.

\section{CONCLUSIONS}

The two experimental examples presented above, along with many others not included here due to space limitations, show the GGMRF algorithm is a promising tool for blind restoration of AO images of space objects. We further conclude that estimated neighborhood influence parameters are appropriate in this class of images for GGMRF blind restoration. Restoration is marginally improved as compared with an arbitrary uniform first-order neighborhood influence parameter matrix. Further work is needed to evaluate algorithm effectiveness for images without dominant edges (e.g. nebula and galaxies) where the blur and image GGMRF models may have similar parameter values.

\section{REFERENCES}

[1] C. Bouman and K. Sauer, "A generalized Gaussian image model for edge-preserving MAP estimation", IEEE Trans. on Image Processing, vol. 2, no. 3, pp. 296-310, July 1993.

[2] S.M. Jefferies and J.C. Christou, "Restoration of astrononmical images by iterative blind deconvolution", Astrophysical Journal, vol. 415, pp. 862-874, Oct. 1993.

[3] J.D. Drummond, "Sizes, shapes and rotational poles of ceres and vesta from adaptive optics images", Bulletin of the American Astronomical Society, vol. 27, pp. 16, 1996.

[4] J.D. Drummond, R.Q. Fugate, J.C. Christou, and E.K. Hege, "Full adaptive optics imgaes of asteroids ceres and vesta: Rotational poles and triaxial ellipsoid dimensions", to appear in ICARUS, 1998.

[5] B.D. Jeffs and J.C. Christou, "Blind bayesian restoration of adaptive optics telescope images using generalized gaussian markov random field models", Proceedings of the SPIE, vol. 3353: Conference on Adaptive Optics and Telescope Systems, Mar. 1998.

[6] Wai Ho Pun, Parametric Model-Adaptive Image Restoration, $\mathrm{PhD}$ thesis, Brigham Young University, 1995.
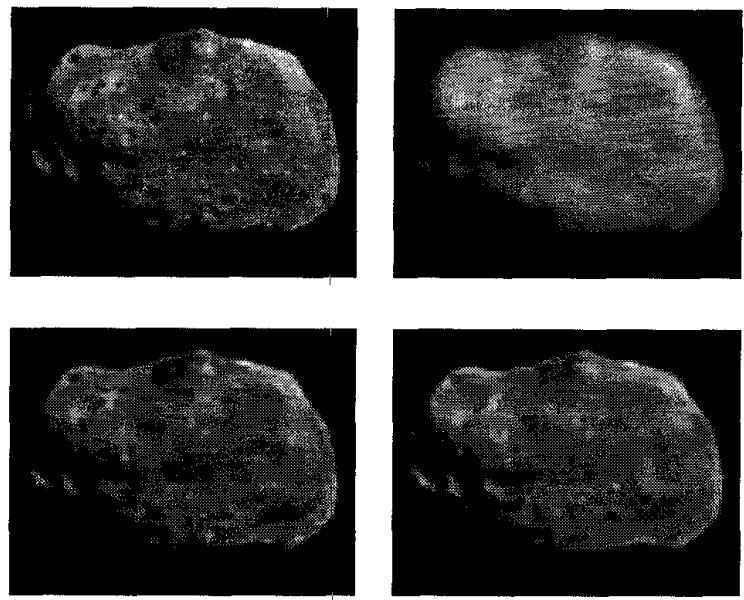

Figure 7. Restoration Results Using Estimated Neighborhood Influence Parameters and Uniform Neighborhood Influence Parameters. a)[Top Left] Original $115 \times 150$ pixel image, b)[Top Right] Single frame of AO residual blur image, c)[Bottom Left] Restoration using estimated neighborhood influence parameter, with $p=1.1, \gamma=4 \times 10^{-4}$ and $\lambda=1.5 \times 10^{-5}$, d)[Bottom Right] Restoration using uniform neighborhood influence parameter, $p=1.1, \gamma=5 \times 10^{-4}$ and $\lambda=2.1 \times 10^{-5}$.

[7] W. H. Pun and B. D. Jeffs, "Shape parameter estimation for generalized gaussian markov random field models used in map image restoration", in Conference Record, 29th Asilomar Conf. Signals, Syst., Comp., Oct. 1995, pp. 1472-1476.

[8] Hong Hong, "Bind bayesian restoration for adaptive optics telescope images", Master's thesis, Brigham Young University, 1998.

[9] R. L. Kashyap and R. Chellappa, "Estimation and choice of neighbors in spatial-interaction models of images", IEEE Transactions on Information Theory, vol. 29, pp. 60-72, Jan. 1983.

[10] J. Besag, "Spatial interaction and the statistical analysis of lattice systems", Journal of the Royal Statistical Society, Series B, vol. 36, pp. 192-236, 1974.

[11] C. C. Chen, Markov random field models in image analysis, PhD thesis, Michigan State University, East Lansing, 1988. 